\title{
Genome-wide alterations in gene methylation by the BRAF V600E mutation in papillary thyroid cancer cells
}

\author{
Peng Hou, Dingxie Liu and Mingzhao Xing
}

Laboratory for Cellular and Molecular Thyroid Research, Division of Endocrinology and Metabolism, The Johns Hopkins University School of Medicine, 1830 East Monument Street, Suite 333, Baltimore, Maryland 21287, USA

(Correspondence should be addressed to M Xing; Email: mxing1@jhmi.edu)

\begin{abstract}
The BRAFV600E mutation plays an important role in the tumorigenesis of papillary thyroid cancer (PTC). To explore an epigenetic mechanism involved in this process, we performed a genomewide DNA methylation analysis using a methylated $\mathrm{CpG}$ island amplification (MCA)/CpG island microarray system to examine gene methylation alterations after shRNA knockdown of BRAF $\mathrm{V} 600 \mathrm{E}$ in thyroid cancer cells. Our results revealed numerous methylation targets of BRAF V600E mutation with a large cohort of hyper- or hypo-methylated genes in thyroid cancer cells, which are known to have important metabolic and cellular functions. As hypomethylation of numerous genes by $B R A F$ V600E was particularly a striking finding, we took a further step to examine the selected 59 genes that became hypermethylated in both cell lines upon BRAF V600E knockdown and found them to be mostly correspondingly under-expressed (i.e. they were normally maintained hypomethylated and over-expressed by BRAF V600E in thyroid cancer cells). We confirmed the methylation status of selected genes revealed on MCA/CpG microarray analysis by performing methylation-specific PCR. To provide proof of concept that some of the genes uncovered here may play a direct oncogenic role, we selected six of them to perform shRNA knockdown and examined its effect on cellular functions. Our results demonstrated that the HMGB2 gene played a role in PTC cell proliferation and the FDG1 gene in cell invasion. Thus, this study uncovered a prominent epigenetic mechanism through which BRAF V600E can promote PTC tumorigenesis by altering the methylation and hence the expression of numerous important genes.
\end{abstract}

Endocrine-Related Cancer (2011) 18 687-697

\section{Introduction}

Papillary thyroid cancer (PTC) is the most common endocrine malignancy, accounting for $80 \%$ of all thyroid cancers (Hundahl et al. 1998, Altekruse et al. 2010). A strong oncogenic mutation that has been characterized in recent years in PTC is the $B R A F$ mutation, which, by far, is the most common oncogenic genetic event found in this cancer, occurring in about $45 \%$ of cases on average (Xing 2005). There are several types of BRAF mutations found in PTC and the T1799A transverse point mutation accounts for vast majority of them (Nikiforov 2008). The T1799A BRAF mutation causes a substitution of valine with glutamic acid in codon 600 (V600E), resulting in constitutive and oncogenic activation of the BRAF kinase in the Ras/Raf/MEK/ERK signaling pathway (MAPK pathway; Davies et al. 2002). Through activating the MAPK pathway, the $B R A F$ mutation plays a fundamental role in the tumorigenesis of PTC and promotes and predicts its poor clinical outcomes (Xing 2005, 2007a). The specific molecular mechanisms underlying PTC tumorigenesis driven by the BRAF mutation have not been well understood. This is particularly the case in epigenetic aspects. For example, the role of aberrant gene methylation and its extent in this process have not been defined for BRAF mutation in PTC.

Gene methylation is an epigenetic phenomenon in which a methyl group is covalently added to the fifth 
carbon of the cytosine residue in a $\mathrm{CpG}$ dinucleotide in $\mathrm{CpG}$ islands, typically located in the promoter area of a gene. Promoter methylation often silences a gene and aberration in its methylation state can thus seriously affect its function. As such, hypermethylation can silence tumor suppressor genes and hypomethylation can cause over-expression of oncogenes. Consequently, aberrant alterations in gene methylation play a fundamental role in human tumorigenesis (Jones \& Baylin 2007, Hsiao et al. 2009, Kondo \& Issa 2010), including thyroid tumorigenesis (Xing 2007b).

Given the importance of this epigenetic aberration in thyroid tumorigenesis, we hypothesize that the $B R A F$ V600E mutation, through activating the MAPK pathway, may aberrantly affect gene methylation in thyroid cancer, as a molecular mechanism in $B R A F$ mutation-promoted thyroid tumorigenesis. In this study, we performed a genome-wide screening of gene methylation in thyroid cancer cells using a methylated $\mathrm{CpG}$ island amplification (MCA)/CpG island microarray approach (Estécio et al. 2007) and selectively examined the cellular functions of some genes among which many identified to be altered in the methylation state by $B R A F$ V600E.

\section{Materials and methods}

\section{Thyroid cancer cell lines}

The PTC-derived cell lines BCPAP and OCUT1 were from Dr Massimo Santoro (University of Federico II, Naples, Italy) and Dr Naoyoshi Onoda (Osaka City University Graduate School of Medicine, Osaka, Japan) respectively. We chose these two thyroid cancer cell lines because both harbor the $B R A F$ V600E mutation. Cells were routinely grown at $37^{\circ} \mathrm{C}$ in RPMI 1640 medium containing $10 \%$ fetal bovine serum (FBS) with standard supplements. Genomic DNA was isolated by SDS and proteinase K digestion, followed by standard phenol-chloroform extraction and ethanol precipitation.

\section{Lentivirus-mediated RNA interference}

The lentiviral pSicoR-PGK-puro vectors (Addgene, Inc., Cambridge, MA, USA) encoding seven selfcomplementary hairpin RNA sequences were used to knock down seven selected genes, including BRAF, HLX1, KLHL14, HMGB2, NR4A2, FGD1, and ZBTB10. The sequences are presented in Supplementary Table 1, see section on supplementary data given at the end of this article. Lentiviral vector DNAs and packaging vectors were transfected into human embryonickidney293cells(ATCC,Manassas,VA,USA) to generate lentiviral particles. Cells were then exposed to lentivirus-containing supernatant for $48 \mathrm{~h}$ in the presence of polybrene $(8 \mu \mathrm{g} / \mathrm{ml}$ final concentration). After 2 days, transfected cells were serum starved (0.5\% FBS) and then harvested for $24 \mathrm{~h}$ in RIPA lysis buffer (Santa Cruz Biotechnology, Santa Cruz, CA, USA). Western blotting assays were used to detect the protein expression of BRAF, HLX1, KLHL14, HMGB2, NR4A2, FGD1, and ZBTB10. Cell pools with stable transfection were selected using puromycin.

\section{Western blotting assay}

Cells were lysed in RIPA buffer using standard protocol. Protein lysates were separated on 10\% SDS-PAGE and transferred onto polyvinylidene fluoride (PVDF) membranes (Amersham Pharmacia Biotech), followed by incubation with specific primary antibodies. Anti-BRAF (sc-166), anti-phospho-ERK (sc-7383), anti-ZBTB10 (sc-87402), and anti-actin (sc-1616-R) were purchased from Santa Cruz (Santa Cruz, CA, USA). Anti-HLX1 (AV100839), anti-KLHL14 (AV34677), anti-HMGB2 (AV31939), anti-NR4A2 (AV38753), and anti-FGD1 (AV31673) were purchased from Sigma. Antigenantibody complexes were visualized using HRP-conjugated anti-mouse (sc-2005, Santa Cruz) or anti-rabbit (sc-2004, Santa Cruz) IgG antibodies and ECL western blotting analysis system (Amersham Pharmacia).

\section{Methylated CpG island amplification}

The MCA assay was performed as described previously (Toyota et al. 1999). Briefly, $\sim 5 \mu \mathrm{g}$ of each genomic DNA from cells stably transfected with BRAF shRNA (termed 'test' hereafter) and vector (termed 'control' hereafter) were digested with 100 units of methylation-sensitive restriction endonuclease SmaI (New England Biolabs, Ipswich, MA, USA) for $16 \mathrm{~h}$ at $20^{\circ} \mathrm{C}$ in a total volume of $100 \mu \mathrm{l}$, followed by digestion with 20 units of methylation-insensitive restriction endonuclease $\mathrm{XmaI}$ for $6 \mathrm{~h}$ at $37^{\circ} \mathrm{C}$, which leaves sticky ends (C/CCGGG). DNA fragments were then precipitated with ethanol and their sticky ends ligated with $0.5 \mathrm{nmol}$ of unphosphorylated linkers RXMA - 24/-12, as described (Toyota et al. 1999). The oligonucleotide sequences were as follows: (RXMA-24) 5'-AGC ACT CTC CAG CCT CTC ACC GAC-3' and (RXMA-12) 5'-CCG GGT CGG TGA-3'. PCR reactions were performed using the digests as templates and subjected to 20 cycles of amplification as described previously (Estécio et al. 2007). Ten microliters of PCR product were resolved in $1.5 \%$ agarose gel and visualized under u.v. after ethidium bromide staining. Successful MCA reactions 
resulted in amplicon smear ranging from $300 \mathrm{bp}$ to $3 \mathrm{~kb}$, with most amplicons at $1 \mathrm{~kb}$. The PCR products were purified and reserved for fluorescence labeling.

\section{CpG island microarray}

Amino-allyl dUTP (aa-dUTP; Sigma) was incorporated into $600 \mathrm{ng}$ of 'test and control' amplicons using the BioPrime DNA labeling system (Life Technologies, Inc., Carlsbad, CA, USA). Alexa 647 (red color) and Alexa 555 (green color) fluorescent dyes were coupled to aa-dUTP-labeled 'test and control' amplicons respectively. Equimolar amounts of the labeled amplicons were co-hybridized to the $12 \mathrm{~K}$ Human CpG-island Array chip (Microarray Center, University Health Network, Toronto, ON, Canada), which contained $12192 \mathrm{CpG}$-island clones that were originally created at the Sanger Institute (Heisler et al. 2005). Hybridized slides were scanned and the acquired images were analyzed with the corresponding software. Two-step global lowess normalization was done using the background-subtracted median intensity of each spot, and the resultant $\log _{2}$ ratios were averaged from triplicate experiments. $\mathrm{CpG}$ island tags (or probes) hybridized predominantly with the test amplicon, but not with the control amplicon, appear as red spots. We set an arbitrary cutoff value of 1.5 for the Alexa 647/555 ratio as previously defined (Yan et al. 2001, Shi et al. 2002); loci with ratios $\geq 1.5$ (equally $\log _{2}$ ratio $\geq 0.6$ ) were identified as hypermethylated in the 'test' samples. In contrast, $\mathrm{CpG}$ island tags hybridized predominantly with the 'control' amplicon, but not with the test amplicon, appear as green spots. We set a cutoff value of 0.5 for the Alexa $647 / 555$ ratio; loci with ratios $\leq 0.5$ (equally $\log _{2}$ ratio $\leq-1$ ) were identified as hypomethylated in the 'test' samples (Yan et al. 2001, Shi et al. 2002). Triplicate experiments were performed for each cell line and they were averaged for data analysis.

\section{Pathway and functional analyses of the hypermethylated genes with Ingenuity Pathways Analysis}

Genes were uploaded to Ingenuity Pathways Analysis (IPA; Ingenuity Systems, Inc., Redwood City, CA, USA; www.ingenuity.com) and pathway and functional analyses were performed. IPA is a commercial web-based interface that uses various computational algorithms to identify and establish cellular networks. This analysis uses a database of gene interactions culled from the literature and updated every quarter of the year.

\section{RNA extraction and real-time quantitative RT-PCR analysis}

RNA expression analysis was performed by real-time quantitative PCR using the ABI Prism 7900HT Sequence Detection System Instrument (Applied Biosystems, Carlsbad, CA, USA). Two micrograms of total RNA underwent RT using Oligo-dT and SuperScript II according to the instructions of the manufacturer (SuperScript First-Strand Synthesis kit, Invitrogen). PCR reactions were performed using SYBR GreenER qPCR SuperMix according to the instructions of the manufacturer (Applied Biosystems). PCR primers were designed using Primer Express (Applied Biosystems) to span a large intron whose location and sequences are presented in Supplementary Table 2, see section on supplementary data given at the end of this article. $C_{\mathrm{t}}$ values were calculated by the ABI PRISM Software and the relative amount of RNA was presented for each sample according to the $2^{-\Delta \Delta C_{t}}$ method (Livak \& Schmittgen 2001). The specificity of real-time quantitative PCR of samples was confirmed by running the PCR products on $1.5 \%$ agarose gel to confirm single specific bands at the expected sizes (data not shown). Each sample was run in triplicate.

\section{Quantitative methylation-specific PCR}

Genomic DNA was subjected to bisulfite treatment as described previously (Hou et al. 2008). A methylationspecific and SYBR-green-based quantitative PCR (QMSP; Hattermann et al. 2008) was performed to validate the methylation status of selected 11 genes on an ABI Prism 7900HT Sequence Detection System (Applied Biosystems) using iTaq SYBR Green Supermix (Bio-Rad) according to the instructions of the manufacturer, including NRA4A2, HLX1, KLHL14, HMGB2, FGD1, ZBTB10, KIAA0247, SLC5A1, TBC1D7, SLMO1, and UCK2. The methylationspecific primers were designed using Methyl Primer Express Software v1.0 (Applied Biosystems) and are presented in Supplementary Table 3, see section on supplementary data given at the end of this article. Normal leukocyte DNA was methylated in vitro with Sss I methylase (New England Biolabs) to generate completely methylated DNA as a positive control. Each plate contained triplicate samples, multiple water blanks, and serial dilutions of positive methylated control to construct the standard curves. The relative methylation level of each DNA sample was calculated as described previously (Hu et al. 2006). 


\section{Cell proliferation and invasion assays}

For cell proliferation assay, cells (800/well) were seeded onto 96-well plates and cultured with $2.5 \%$ FBS. MTT assay was performed daily over a 5-day time course to evaluate cell proliferation. Cell culture was added with $10 \mu \mathrm{l}$ of $5 \mathrm{mg} / \mathrm{ml}$ MTT agent (Sigma) and incubated for $4 \mathrm{~h}$, followed by the addition of $100 \mu \mathrm{l}$ of $10 \%$ SDS solution and a further incubation overnight. The plates were read on a microplate reader using the test wavelength of $570 \mathrm{~nm}$ and the reference wavelength of $670 \mathrm{~nm}$. Three triplicates were done to determine each data point.

Cell invasion assay was performed using Matrigelcoated Transwell cell culture chambers with $8 \mu \mathrm{m}$ pore (BD Biosciences, Sparks, MD, USA). Briefly, $2.5 \times 10^{4}$ cells were suspended in serum-free medium and seeded in the upper chamber of the Transwell insert. RPMI 1640 medium containing 10\% FBS was added to the lower chamber. After a $22 \mathrm{~h}$ incubation at $37{ }^{\circ} \mathrm{C}$ with $5 \% \mathrm{CO}_{2}$, non-invasive cells in the upper chamber were removed and invasive cells that migrated to the underside of the membrane were fixed in $100 \%$ methanol and stained with $0.5 \%$ crystal violet in $2 \%$ ethanol. The number of invasive cells was counted after photography.

\section{Statistical analysis}

Experiments were done at least twice. DNA methylation and gene expression data were analyzed using program ScanAlyze as described by Michael Eisen (http://rana.lbl.gov/eisensoftware.htm). Data were compared using the $t$-test. Statistical significance was defined as $P<0.05$.

\section{Results}

\section{Genome-wide identification of hyper- or hypo- methylated genes by the BRAF V600E mutation in thyroid cancer cells}

To explore the relationship between BRAF V600E mutation and aberrant DNA methylation and identify the methylation targets of the $B R A F$ mutation, we used the shRNA approach to specifically knock down BRAF in thyroid cancer cell lines, BCPAP and OCUT1,

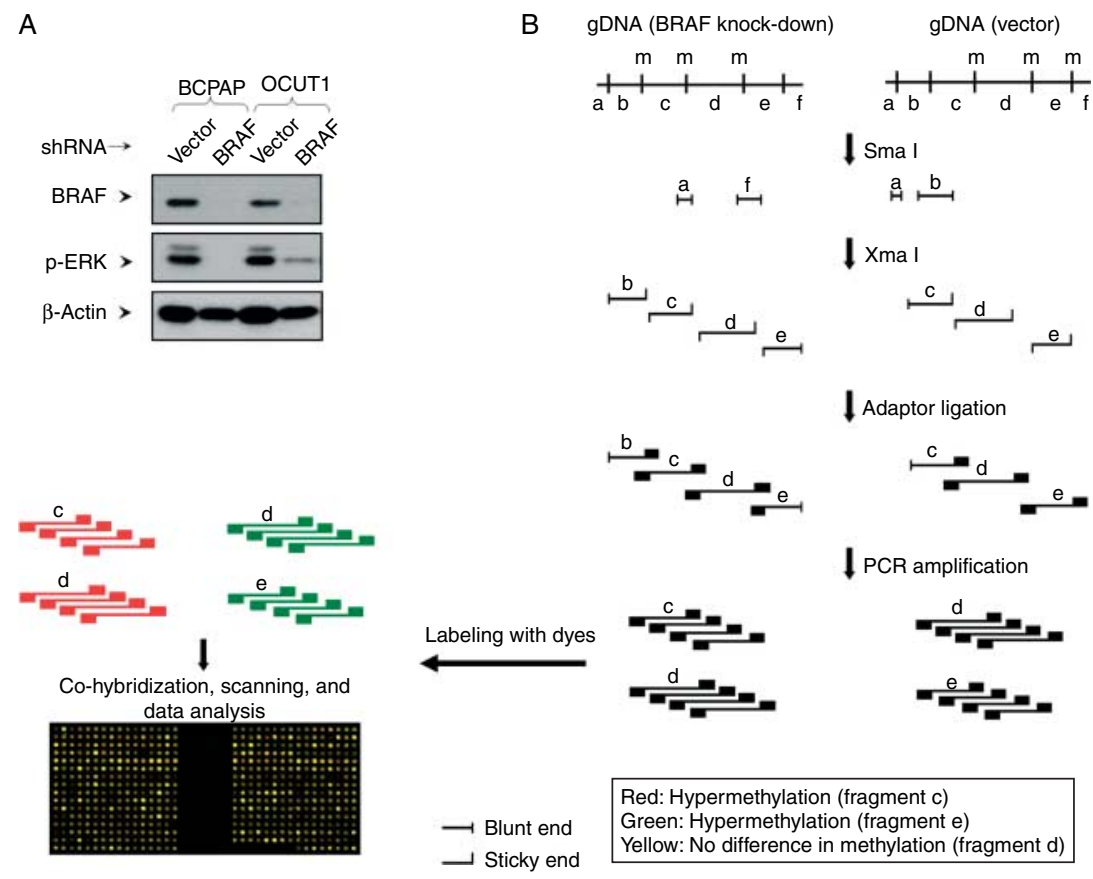

Figure 1 Global identification of $B R A F$ V600E mutation-mediated methylation targets in thyroid cancer cells using the MCA/CpG island microarray approach. (A) Effects of stable siRNA knockdown of BRAF on the MAPK pathway in thyroid cancer cells harboring the BRAF V600E mutation. BCPAP and OCUT1 cells were infected with lentiviruses carrying BRAF shRNA and stable populations were selected with $2 \mu \mathrm{g} / \mathrm{ml}$ puromycin. The empty vector was used as the control. After a 2-week selection, cells were lysed and immunoblotted with BRAF and phosphorylated ERK ( $p$-ERK) antibodies. The antibody against $\beta$-actin was used for quality control of western blotting. (B) Schematic illustration of MCA/CpG island microarray analysis. Methylated $\mathrm{CpG}$ islands were amplified by serial digestion with Smal (methylation sensitive) and Xmal (methylation insensitive) restriction endonucleases. The methylated fraction of genomic DNA from cells stably transfected with BRAF shRNA or vector was amplified and labeled with appropriate dyes and then co-hybridized to a microarray platform as described in the Materials and methods section. 
which harbored $B R A F$ V600E, and examined globally the change in the methylation status of genes using the $\mathrm{MCA} / \mathrm{CpG}$ island microarray approach. As shown in Fig. 1A, BRAF shRNA nearly completely eliminated the expression of BRAF protein. This effectively suppressed the signaling of the MAPK pathway, as reflected by the elimination of phosphorylation of ERK in the two cells. Hypermethylated and hypomethylated genes in 'test' were visualized as red and green spots, respectively, in the microarray. Normalized $\log _{2}$ ratios of $\geq 0.6$ and $\leq-1$ were used as the cutoff values for hypermethylation and hypomethylation, respectively, as conventionally used. A schematic illustration of the procedure and partial $\mathrm{MCA} / \mathrm{CpG}$ island microarray image are presented in Fig. 1B.

A

B
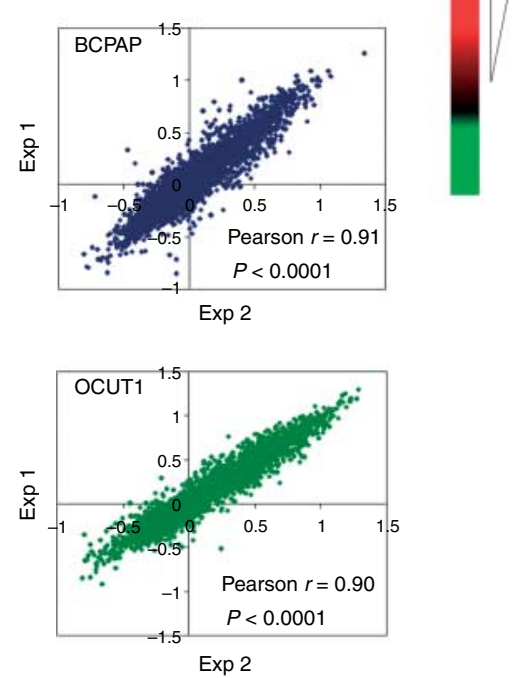

$\operatorname{Exp} 2$

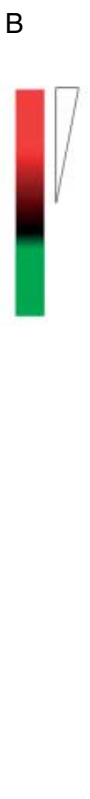

The correlation between two different microarray experiments was statistically significant $(P<0.0001)$, with Pearson $r$ values of 0.91 and 0.90 for cells BCPAP and OCUT1, respectively, suggesting that the $\mathrm{MCA} / \mathrm{CpG}$ island microarray used to identify hypermethylated or hypomethylated genes in this study was highly reproducible and reliable (Fig. 2A). As shown in Fig. 2B, we found a similar methylation profiling in the two thyroid cancer cells after BRAF knockdown. Strikingly, using the corresponding gene database (University Health Network Human CpG Microarray Database, Toronto, ON, Canada; http://data.microarrays. ca/cpg) to identify the specifically affected genes, this approach revealed numerous hyper- or hypo-methylated genes by the BRAF V600E. With a cutoff value of

Figure 2 Detection of gene methylation changes by the MCA/CpG island microarray analysis. (A) Demonstration of the reproducibility of $\mathrm{MCA} / \mathrm{CpG}$ island microarray performance, showing overall correlation between independent replicates for the two thyroid cancer cell lines BCPAP and OCUT1. (B) Global methylation analysis of thyroid cancer cells by the MCA/CpG island microarray analysis. Data are presented as the mean of values from three experiments for the ratios of methylation presented as $\log _{2}$, with red representing genes with high methylation, black representing no change, and green representing hypomethylation. The upper frame indicates 59 representative genes that became hypermethylated upon BRAF V600E knockdown in both BCPAP and OCUT1 cells, which were further analyzed for gene expression, which is shown in Fig. 3 . The lower frame indicates ten representative genes that became hypomethylated upon BRAF V600E knockdown in both BCPAP and OCUT1 cells. 
$\log _{2} \leq-1$ as hypomethylation, we found a large cohort of genes that became hypomethylated upon BRAF knockdown in both cell lines (Fig. 2B). This result suggested that these genes were normally hypermethylated under the pressure of BRAF V600E signaling in the two cells. Similarly, with a cutoff value of $\log _{2} \geq 0.6$ as hypermethylation, we identified a large cohort of genes that became hypermethylated upon BRAF knockdown in both cell lines. This result suggested that these genes were normally hypomethylated under the pressure of $B R A F$ V600E signaling in the two cells. Compared with gene hypermethylation, gene hypomethylation has been relatively poorly investigated in human cancers. The present finding of wide gene hypomethylation by $B R A F$ mutation in thyroid cancer cells was thus particularly interesting. We therefore selected 59 (shown in Fig. 2B) of these genes, which contain two or more SmaI/XmaI sites (CCCGGG) in their promoters, for gene expression analyses and some of them for functional studies as presented in the following sections. A summary of the known main functions of these genes is presented in
Supplementary Table 4, see section on supplementary data given at the end of this article.

\section{Expression analysis of the genes that became hypermethylated upon BRAF V600E knockdown in thyroid cancer cells}

We examined the expression status of 59 genes that became hypermethylated upon BRAF knockdown in the two cells (Fig. 2B). This gene expression analysis demonstrated the functional consequence of methylation changes in genes revealed by the $\mathrm{MCA} / \mathrm{CpG}$ microarray analysis. Our results showed that most of these genes were under-expressed (indicated by green color) in the two thyroid cancer cells (Fig. 3A), consistent with their hypermethylation status upon BRAF knockdown (Fig. 2B). Many of these genes showed identical hypoexpression patterns in the two thyroid cancer cells (i.e. genes in green color in both cells in Fig. 3A). These results are consistent with the expectation that hypermethylation of genes typically leads to their silencing. It should be noted that these results from BRAF knockdown paradoxically
A

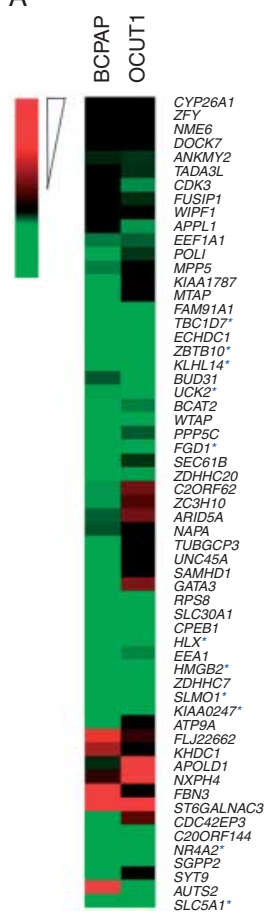

B
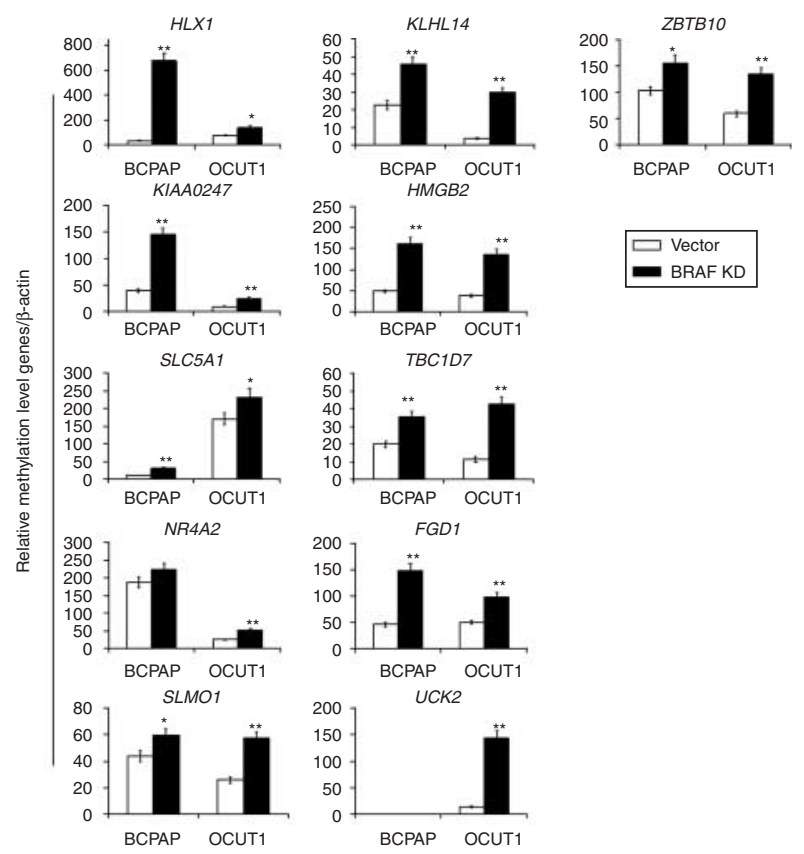

Figure 3 Analysis of gene expression and validation of MCA/CpG island microarray using MSQP in thyroid cancer cells. (A) Gene expression analysis of selected 59 genes from Fig. 2 in BCPAP and OCUT1 cells using RT-PCR, with red color representing overexpression and green color representing under-expression upon BRAF V600E knockdown. (B) Validation by QMSP of methylation status of selected 11 genes that were hypermethylated and under-expressed upon BRAF knockdown in cells revealed by MCA/cpG island microarray. These genes were labeled with an asterisk $\left(^{*}\right)$ in Fig. $3 A$. Details are described in the Materials and methods section. ${ }^{\star} P<0.05$, ${ }^{* \star} P<0.01$. 
demonstrated that these genes were normally hypomethylated and over-expressed in thyroid cancer cells under the pressure of the BRAF V600E mutation.

\section{Validation of hypermethylation of genes after BRAF knockdown in thyroid cancer cells using QMSP}

We selected 11 genes from those that showed hypermethylation by $\mathrm{MCA} / \mathrm{CpG}$ island microarray and hypoexpression in both thyroid cancer cells for methylation validation using QMSP. These genes included NRA4A2, HLX1, KLHL14, HMGB2, FGD1, ZBTB10, KIAA0247, SLC5A1, TBC1D7, SLMO1, and $U C K 2$. As shown in Fig. 3B, suppression of the MAPK signaling pathway through BRAF knockdown could increase methylation level of these genes in the two thyroid cancer cells. These results were consistent with the $\mathrm{MCA} / \mathrm{CpG}$ microarray and gene expression data (Figs $2 \mathrm{~B}$ and $3 \mathrm{~A}$ ), demonstrating again the reliability of the MCA/CpG microarray approach and confirming the true methylation changes in their promoter areas.

\section{Pathway analysis of the genes hypermethylated upon BRAF knockdown}

We used the IPA Software to map the genes that became hypermethylated upon BRAF knockdown to different networks in the IPA database. We chose the top four networks in this study (Table 1). These networks describe functional relationships among gene products based on the findings presented in peerreviewed biological pathways. Genes clustered in these networks are involved in various biological functions, including tissue development, cellular development, carbohydrate metabolism, cell death, DNA replication,

Table 1 Ingenuity networks: the genes hypermethylated upon BRAF V600E knockdown in thyroid cancer cells discovered in this study and involved in the network are presented in bold text

\begin{tabular}{|c|c|c|c|c|}
\hline $\begin{array}{l}\text { Top } \\
\text { network }\end{array}$ & Molecules in network & Score & $\begin{array}{l}\text { Focus } \\
\text { molecules }\end{array}$ & Functions \\
\hline 1 & $\begin{array}{l}\text { APOL3 (includes EG: 80833), AS3MT, AUTS2 } \\
\text { (includes EG: 26053), BCAT2, C14ORF106, } \\
\text { C1ORF123, CASP3, CBLL1, CD40LG, CDH1, } \\
\text { CDK3, CDKN1A, DBNL, ECD, ELMOD3, } \\
\text { FGD1, GATA3, HLX, HNF4A, IFT122, IL12 } \\
\text { (complex), interferon } \alpha, \text { MAP3K14, MPP5, } \\
\text { NAPA, NEURL4, NR3C1, PRR3, RPS8, } \\
\text { SAMHD1, SLC5A1, TADA3, UNC45A, WTAP, } \\
\text { ZC3H10 }\end{array}$ & 36 & 16 & $\begin{array}{l}\text { Tissue development, cellular } \\
\text { development, and cell death }\end{array}$ \\
\hline 2 & $\begin{array}{l}\text { Amino acids, ARID5A, B3GALT2, BUD31 } \\
\text { (includes EG: 8896), C21ORF33, CDC42EP3, } \\
\text { CSRP1, DOCK7 (includes EG: 85440), EEA1, } \\
\text { EGR2, HMGB2, LSR, MN1, NAB1, NDST1, } \\
\text { NFKBIA, NME6, NXPH4, PARP3, PDE7A, } \\
\text { PPP5C, RABGGTA, SEC61B, SGPP2, } \\
\text { sphingosine-1-phosphate, ST6GALNAC3, } \\
\text { STK17A, TBC1D7, TGFB1, TNFSF11, TSC1, } \\
\text { TSC2, Tsc1-Tsc2, UCK2, YWHAA }\end{array}$ & 30 & 14 & $\begin{array}{l}\text { Cellular development, nervous } \\
\text { system development and function, } \\
\text { and carbohydrate metabolism }\end{array}$ \\
\hline 3 & $\begin{array}{l}\text { ANKRA2, APOLD1, APP, ATP9A, CD5L, } \\
\text { CPEB1, CREB1, Cu+, CYP26A1, DUSP14, } \\
\text { FSH, GM10681, GTPASE, hCG, hydrogen } \\
\text { peroxide, KIAA0247, MAP3K5 (includes EG: } \\
\text { 293015), MCL1, NOS3, NOX5, octopamine, } \\
\text { PAPD7, PCNA, POLD4, POLE, POLI, POLK, } \\
\text { POLM, POLQ, PTPase, SFRS13A, SLC30A1, } \\
\text { UNG, WIPF1, ZDHHC7 }\end{array}$ & 19 & 10 & $\begin{array}{l}\text { DNA replication, recombination, and } \\
\text { repair, cellular compromise, and } \\
\text { cellular assembly and organization }\end{array}$ \\
\hline 4 & $\begin{array}{l}\text { ADIPOR1, ADIPOR2, AKT3, ANP32A, APPL1, } \\
\text { ATP5B, CCT2, COL4A1, CSH1, DUSP4, EEF2, } \\
\text { EEF1A1, EIF3F, GPX1, GRB14, Insulin, } \\
\text { KCNMA1, LEPR, MDH2, MTAP (includes EG: } \\
\text { 4507), NR4A2, NR4A3, PGK1, PHYHIP, } \\
\text { PSMD11, Rab5, RPS16, SLC6A3, SP4, SYT9, } \\
\text { TRPV1, TUBGCP3, Vegf, ZBTB10, ZNF24 } \\
\text { (includes EG: 7572) }\end{array}$ & 13 & 7 & $\begin{array}{l}\text { Organismal survival, inflammatory } \\
\text { response, and cell cycle }\end{array}$ \\
\hline
\end{tabular}


recombination, and repair, and other functions. Based on a score of 13 or higher in the top 1-4 networks, many genes, including the 11 genes that are specially studied as described above, that became hypermethylated upon BRAF V600E knockdown in the two thyroid cancer cells showed an extensive relationship and interaction among themselves in the pathways. Thus, these results strongly suggest that many of the genes aberrantly altered in methylation and hence expression by $B R A F$ V600E had important functions in thyroid cancer cells.

\section{Testing of the role in cellular functions of PTC cells of selected genes that were normally hypomethylated and over-expressed under the pressure of BRAF V600E}

We speculated that some of the genes that were hypomethylated and over-expressed by BRAF V600E might play a direct role in cellular functions of thyroid cancer cells. From the 11 genes shown in the pathway/networks revealed by IPA, we chose six genes for such studies in BCPAP cells. These included
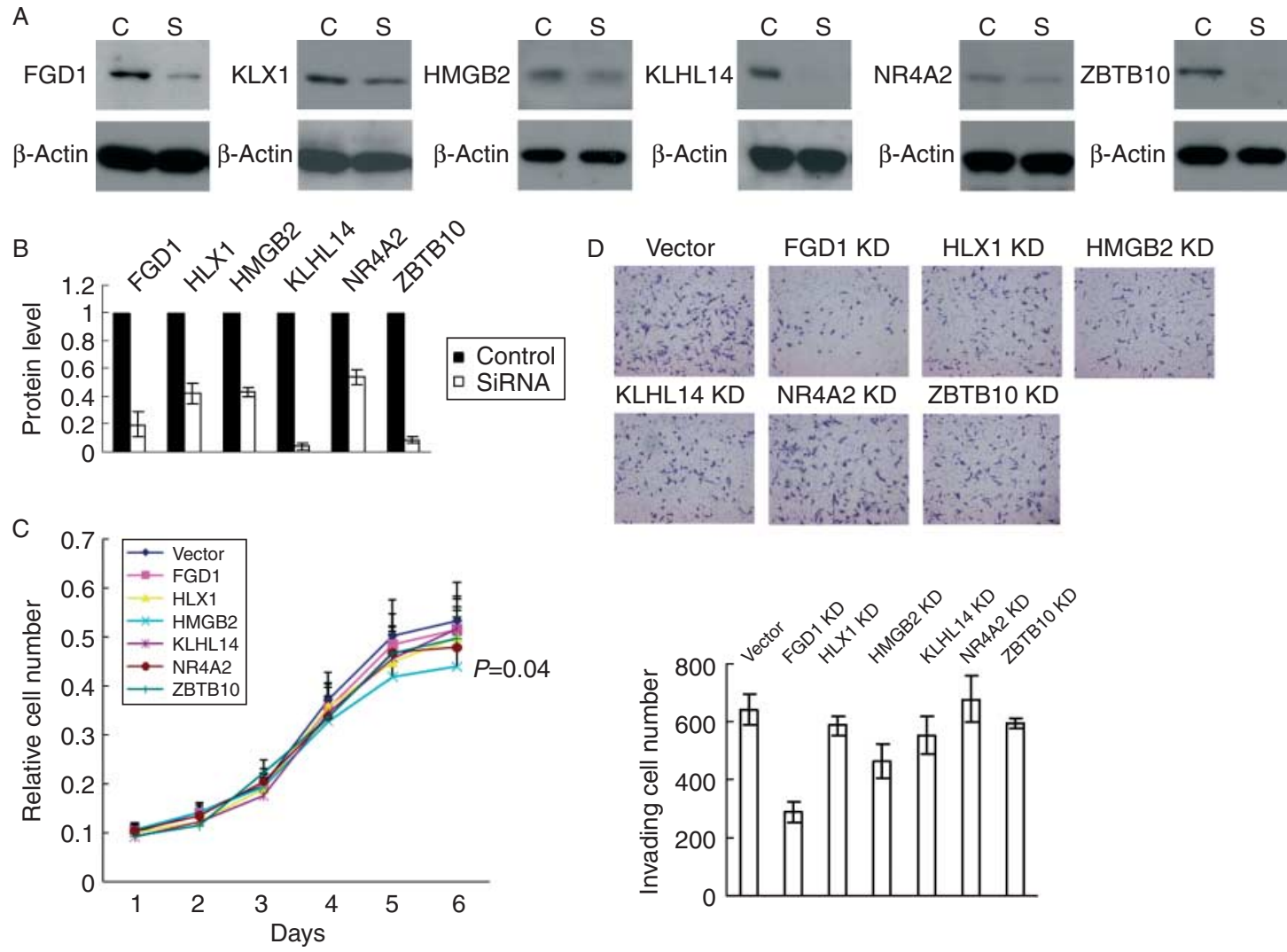

Figure 4 Effects of silencing BRAF V600E-related genes on the proliferation and invasion of thyroid cancer cells. (A) Knock down of the indicated genes coupled to the BRAF V600E signaling through aberrant gene methylation revealed in this study using vectorbased RNA interference. BCPAP cells were infected with lentivirus expressing shRNA targeting FGD1, HLX1, HMGB2, KLHL14, NR4A2, and ZBTB10 respectively. Stable cell pools obtained after selection with puromycin were lysed and immunoblotted with corresponding antibodies. The lentivirus packaged with empty vector was used as control. (B) Bar graph, corresponding to (A), shows the average protein levels of control or shRNA-transfected cells from two independent experiments. The ratio between band intensities of specific proteins and the corresponding $\beta$-actin controls was calculated to correct for differences in protein loading on the gel. For convenient comparison, the protein levels of the control vector-transfected cells were set as 1 and the protein levels of shRNA-transfected cells were set relative to the control for each in indicated gene. The error bars represent two protein levels obtained in the two different experiments. (C) Cell proliferation assay of BCPAP cell pools with stable knock down of the indicated $B R A F$ V600E-coupled genes. The proliferation rate was examined using MTT assay and the OD value was measured daily over a 5 -day time course. Cell numbers were significantly different $(P=0.04)$ at day 6 between the group transfected with vector and the group transfected with HMGB2 shRNA. (D) Cell invasion assay of BCPAP cell pools with stable knock down of the indicated BRAF V600E-related genes. Cells were plated and cultured in Matrigel-coated transwell for $22 \mathrm{~h}$, followed by examination and quantification of the invasive cells as described in the Materials and methods section. The upper panel shows representative results of invasive BCPAP cells with different transfections. The bar graph in the lower panel, corresponding to the upper panel, shows the average number of invasive cells of two independent experiments, with the error bars representing the invasive cell numbers obtained from the two different experiments. C, control shRNA; S, specific shRNA; KD, knockdown. 
FGD1, HLX1, HMGB2, KLHL14, NR4A2, and ZBTB10 genes. To this end, we used the shRNA approach to specifically knock down these genes (Fig. 4A and B) and subsequently examined the change in cellular behaviors of BCPAP cells. Among these six genes, shRNA knockdown of the HMGB2 gene could significantly inhibit the proliferation of BCPAP cells in comparison with control vector transfection $(P=0.04$; Fig. 4C). shRNA knockdown of the FGDI gene could dramatically inhibit the invasion of BCPAP cells (Fig. 4D). These results provide direct evidence that, as representatives of the genes coupled to $B R A F$ mutation through epigenetic alterations, the $H M G B 2$ and $F G D 1$ genes may have an important role in $B R A F$ mutation-promoted thyroid tumorigenesis.

\section{Discussion}

Numerous recent studies have established the important role of $B R A F \mathrm{~V} 600 \mathrm{E}$ mutation in the tumorigenesis and pathogenesis of PTC (Xing 2007a, Nikiforov 2008). However, information on the molecular mechanisms and derangements coupled to this mutation in this process is limited. As methylation aberrations of genes and consequent alterations in their expression are a fundamental molecular mechanism in the tumorigenesis of human cancers (Jones \& Baylin 2007, Hsiao et al. 2009, Kondo \& Issa 2010), including thyroid cancer (Xing 2007b), we explored such a mechanism in the tumorigenesis of PTC promoted by the $B R A F$ mutation in this study.

We took the strategy of using a high-throughput $\mathrm{MCA} / \mathrm{CpG}$ island microarray system to explore genome-wide aberrations of gene methylation coupled to the BRAF mutation in PTC cells. With shRNA knockdown of the BRAF V600E in thyroid cancer cells that naturally harbored this mutation, we examined the change in gene methylation and found, strikingly, a large number of genes to become hyper- or hypomethylated upon knock down of $B R A F$ V600E in PTC cells. It should be noted that under these conditions, the hyper- or hypo-methylated genes represent, paradoxically, hypo- or hyper-methylated genes naturally existing under the pressure of BRAF V600E in these PTC cells. Therefore, a striking finding of this study is that numerous genes are normally hypermethylated or hypomethylated by the MAPK signaling driven by BRAF V600E in PTC cells. Since methylation usually silences a gene, the expression of these genes must be significantly affected by alterations in their methylation states promoted by the $B R A F$ mutation. Many of these genes are already known to play various critical metabolic, molecular, and cellular functions, as also suggested by our IPA pathway analysis for some of these genes. Changes in the expression of these genes driven by the $B R A F$ mutation must have a significant impact on the functions and behaviors of the cell. Thus, this wide aberration of gene methylation in the genome coupled to the BRAF mutation represents a major mechanism that has not been previously revealed in the PTC tumorigenesis promoted by the BRAF mutation.

The gene expression profiling coupled to BRAF V600E was shown to be largely similar between the two thyroid cancer cell lines, BCPAP and OCUT1, in this study, but there are also many genes that are expressed differently between the two cells. This may be due to the difference in other molecular environments of the two cells, such as their different genetic backgrounds; for example, in addition to $B R A F$ mutation, OCUT1 cells also harbor the PIK3CA mutation, which activates the PI3K/Akt pathway.

$B R A F$ mutation was found to be associated with methylation of certain genes in colon cancer (Weisenberger et al. 2006, Ang et al. 2009). A number of genes were previously found to be hypermethylated in thyroid cancer (Xing 2007b) and some tumor suppressor genes were found to be also associated with $B R A F$ mutation in PTC (Hu et al. 2006). However, these association studies did not establish a functional and causal link between the BRAF mutation and the occurrence of methylation of these genes. This study is the first to demonstrate a direct functional link between $B R A F$ mutation and aberrant gene methylation in thyroid cancer cells. Strikingly, this role of $B R A F$ V600E is genome-wide and affects numerous genes, suggesting that epigenetic impact of the BRAF mutation on PTC cells is of paramount importance. By taking a genomewide analysis approach, the finding in this study extended well beyond the scope of previous work on the association of methylation of a few individual genes with $B R A F$ mutation. A few genes previously found to be hypermethylated in association with $B R A F$ mutation in PTC were important tumor suppressor genes (Hu et al. 2006). It is plausible to speculate that many of the new genes implicated to be normally hypermethylated by BRAF V600E signaling in thyroid cancer in this study may have important tumor-suppressing functions, which need to be defined in further studies.

Another novel and striking finding in this study is the numerous genes that are normally hypomethylated under the pressure of $B R A F \mathrm{~V} 600 \mathrm{E}$ in thyroid cancer cells (i.e. the genes that became hypermethylated upon $B R A F$ V600E knockdown). In line with this finding is a recent example that aberrant MAPK signaling pathway driven by the $B R A F$ mutation promoted the expression of $M A P 2$ gene, a neuron-specific 
microtubule-associated protein, in melanoma cells by promoter demethylation (Maddodi et al. 2010). Compared with gene hypermethylation, gene hypomethylation has been relatively under-investigated in cancers in general. We therefore took a further step to investigate this phenomenon in thyroid cancer cells in this study. In particular, we selected 59 genes for expression analysis that contained two or more $\mathrm{SmaI} / \mathrm{XmaI}$ sites in their promoters and were hypermethylated upon BRAF knockdown in the two thyroid cancer cell lines. These selected genes contained two or more SmaI/XmaI sites in their promoters, ensuring that, if identified by the MCA approach used in the present study, they can have methylation alterations in their promoter areas. As expected, most of these genes were correspondingly under-expressed upon BRAF knockdown in the two thyroid cancer cells, consistent with the classically known gene-silencing role of hypermethylation. This result paradoxically suggests that, driven by the $B R A F \mathrm{~V} 600 \mathrm{E}$, these genes are normally over-expressed as well as hypomethylated in PTC cells. We also used QMSP to confirm the methylation states of selected genes revealed by the $\mathrm{MCA} / \mathrm{CpG}$ island microarray with corresponding under-expression in both thyroid cancer cells and were able to validate the $\mathrm{MCA} / \mathrm{CpG}$ island microarray approach in evaluating gene methylation changes.

Similar to the significance of hypermethylation and presumably under-expression of the large cohort of genes coupled to the BRAF $\mathrm{V} 600 \mathrm{E}$ as discussed above, we speculated that hypomethylation and overexpression of the large cohort of genes coupled to this mutant should also have profound effect on cellular function and tumor behavior of PTC. It is tempting to propose that many of these genes coupled to the BRAF V600E through hypomethylation may play an important tumor-promoting role in the PTC tumorigenesis. In fact, our IPA analyses showed that many of the genes coupled to BRAF mutation through hypomethylation are important components of several major pathways involved in various metabolic and cellular functions. It is possible that some of these genes may prove to be novel pro-oncogenes or oncogenes in the future. To functionally and principally test this concept, we selected six genes to study their role in cellular functions of PTC cells. Even among only six genes chosen to study, we were able to demonstrate that two of them played a functional role in PTC cells. Specifically, the FDG1 gene played a significant role in PTC cell invasion and the $H M G B 2$ gene played a role in cell proliferation. These results imply a potential oncogenic function of the FDG1 and HMGB2 genes. The FDG1 gene is the susceptible gene for the Aarskog-Scott's faciogenital dysplasia syndrome (Shalev et al. 2006) and the $H M G B 2$ gene is a DNA-binding protein with pleiotropic biological functions (Yamada \& Maruyama 2007). A previous study in fact showed that expression of the FDGl gene could cause cell transformation (Whitehead et al. 1998). It should be pointed out that the methylation status and consequent biological effects of many genes are not affected by the BRAF V600E signaling as shown in this study, although many are. This is not surprising as other more dominant regulatory mechanisms may be involved in the regulation of these genes. Also, epigenetic alterations could be irreversible under certain molecular and cellular circumstances.

In summary, using a high-throughput $\mathrm{MCA} / \mathrm{CpG}$ island microarray approach, we performed a genomewide gene methylation analysis with respect to $B R A F$ mutation in PTC cells. We uncovered widespread alterations in DNA methylation with a large cohort of hyper- or hypo-methylated genes under the pressure of $B R A F$ V600E signaling. Given the known important metabolic and cellular functions of many of these genes, alterations in their expression through methylation aberrations as a consequence of $B R A F$ mutation may have a profound impact on cell functions and behaviors of PTC. Many of these genes, as suggested by the FDG1 and $H M G B 2$ genes, may prove to play an important role in thyroid tumorigenesis. This study has thus uncovered a prominent epigenetic mechanism through which the BRAF mutation drives the tumorigenesis of PTC.

\section{Supplementary data}

This is linked to the online version of the paper at http://dx. doi.org/10.1530/ERC-11-0212.

\section{Declaration of interest}

The authors declare that there is no conflict of interest that could be perceived as prejudicing the impartiality of the research reported.

\section{Funding}

This work was supported by NIH grant RO-1 CA113507 (to M X).

\section{Acknowledgements}

We thank Drs Massimo Santoro and Naoyoshi Onoda for providing us BCPAP and OCUT1 cells respectively. 


\section{References}

Altekruse SF, Kosary CL, Krapcho M, Neyman N, Aminou R, Waldron W, Ruhl J, Howlader N, Tatalovich Z, Cho H, et al. (eds) 2010. SEER Cancer Statistics Review, 1975-2007. National Cancer Institute: Bethesda, MD, USA, http://seer.cancer.gov/csr/1975_2007/, based on November 2009 SEER data submission, posted to the SEER web site.

Ang PW, Li WQ, Soong R \& Iacopetta B 2009 BRAF mutation is associated with the $\mathrm{CpG}$ island methylator phenotype in colorectal cancer from young patients. Cancer Letters 273 221-224. (doi:10.1016/j.canlet.2008. 08.001)

Davies H, Bignell GR, Cox C, Stephens P, Edkins S, Clegg S, Teague J, Woffendin H, Garnett MJ, Bottomley W et al. 2002 Mutations of the BRAF gene in human cancer. Nature 417 949-954. (doi:10.1038/nature00766)

Estécio MR, Yan PS, Ibrahim AE, Tellez CS, Shen L, Huang TH \& Issa JP 2007 High-throughput methylation profiling by MCA coupled to CpG island microarray. Genome Research 17 1529-1536. (doi:10.1101/gr.6417007)

Hattermann K, Mehdorn HM, Mentlein R, Schultka S \& Held-Feindt J 2008 A methylation-specific and SYBRgreen-based quantitative polymerase chain reaction technique for O6-methylguanine DNA methyltransferase promoter methylation analysis. Analytical Biochemistry 377 62-71. (doi:10.1016/j.ab.2008.03.014)

Heisler LE, Torti D, Boutros PC, Watson J, Chan C, Winegarden N, Takahashi M, Yau P, Huang TH, Farnham PJ et al. $2005 \mathrm{CpG}$ island microarray probe sequences derived from a physical library are representative of CpG islands annotated on the human genome. Nucleic Acids Research 33 2952-2961. (doi:10.1093/nar/ gki582)

Hou P, Ji M \& Xing M 2008 Association of PTEN gene methylation with genetic alterations in the phosphatidylinositol 3-kinase/AKT signaling pathway in thyroid tumors. Cancer 113 2440-2447. (doi:10.1002/cncr.23869)

Hsiao SH, Huang TH \& Leu YW 2009 Excavating relics of DNA methylation changes during the development of neoplasia. Seminars in Cancer Biology 19 198-208. (doi:10.1016/j.semcancer.2009.02.015)

Hu S, Liu D, Tufano RP, Carson KA, Rosenbaum E, Cohen Y, Holt EH, Kiseljak-Vassiliades K, Rhoden KJ, Tolaney S et al. 2006 Association of aberrant methylation of tumor suppressor genes with tumor aggressiveness and BRAF mutation in papillary thyroid cancer. International Journal of Cancer 119 2322-2329. (doi:10.1002/ijc.22110)

Hundahl SA, Fleming ID, Fremgen AM \& Menck HR 1998 A National Cancer Data Base report on 53856 cases of thyroid carcinoma treated in the U.S., 1985-1995. Cancer 83 2638-2648. (doi:10.1002/(SICI)1097-0142(19981215) 83:12<2638::AID-CNCR31>3.0.CO;2-1)

Jones PA \& Baylin SB 2007 The epigenomics of cancer. Cell 128 683-692. (doi:10.1016/j.cell.2007.01.029)

Kondo Y \& Issa JP 2010 DNA methylation profiling in cancer. Expert Reviews in Molecular Medicine 12 e23. (doi:10.1017/S1462399410001559)
Livak KJ \& Schmittgen TD 2001 Analysis of relative gene expression data using real-time quantitative PCR and the 2(-Delta Delta C(T)) method. Methods 25 402-408. (doi:10.1006/meth.2001.1262)

Maddodi N, Bhat KM, Devi S, Zhang SC \& Setaluri V 2010 Oncogenic BRAFV600E induces expression of neuronal differentiation marker MAP2 in melanoma cells by promoter demethylation and down-regulation of transcription repressor HES1. Journal of Biological Chemistry 285 242-254. (doi:10.1074/jbc.M109.068668)

Nikiforov YE 2008 Thyroid carcinoma: molecular pathways and therapeutic targets. Modern Pathology 21 (Suppl 2) S37-S43. (doi:10.1038/modpathol.2008.10)

Shalev SA, Chervinski E, Weiner E, Mazor G, Friez MJ \& Schwartz CE 2006 Clinical variation of Aarskog syndrome in a large family with $2189 \mathrm{del}$ A in the FGD1 gene. American Journal of Medical Genetics. Part A 140 162-165. (doi:10.1002/ajmg.a.31033)

Shi H, Yan PS, Chen CM, Rahmatpanah F, Lofton-Day C, Caldwell CW \& Huang TH 2002 Expressed CpG island sequence tag microarray for dual screening of DNA hypermethylation and gene silencing in cancer cells. Cancer Research 62 3214-3220.

Toyota M, Ho C, Ahuja N, Jair KW, Li Q, Ohe-Toyota M, Baylin SB \& Issa JP 1999 Identification of differentially methylated sequences in colorectal cancer by methylated CpG island amplification. Cancer Research 59 2307-2312.

Weisenberger DJ, Siegmund KD, Campan M, Young J, Long TI, Faasse MA, Kang GH, Widschwendter M, Weener D, Buchanan D et al. 2006 CpG island methylator phenotype underlies sporadic microsatellite instability and is tightly associated with BRAF mutation in colorectal cancer. Nature Genetics 38 787-793. (doi:10.1038/ng1834)

Whitehead IP, Abe K, Gorski JL \& Der CJ 1998 CDC42 and FGD1 cause distinct signaling and transforming activities. Molecular and Cellular Biology 18 4689-4697.

Xing M 2005 BRAF mutation in thyroid cancer. EndocrineRelated Cancer 12 245-262. (doi:10.1677/erc.1.0978)

Xing M 2007a BRAF mutation in papillary thyroid cancer: pathogenic role, molecular bases, and clinical implications. Endocrine Reviews 28 742-762. (doi:10.1210/er.2007-0007)

Xing M $2007 b$ Gene methylation in thyroid tumorigenesis. Endocrinology 148 948-953. (doi:10.1210/en.2006-0927)

Yamada S \& Maruyama I 2007 HMGB1, a novel inflammatory cytokine. Clinica Chimica Acta 375 36-42. (doi:10.1016/j.cca.2006.07.019)

Yan PS, Chen CM, Shi H, Rahmatpanah F, Wei SH, Caldwell CW \& Huang TH 2001 Dissecting complex epigenetic alterations in breast cancer using $\mathrm{CpG}$ island microarrays. Cancer Research 61 8375-8380.

Received in final form 20 September 2011

Accepted 21 September 2011

Made available online as an Accepted Preprint 21 September 2011 